\title{
Avaliação do efeito analgésico preemptivo do lumiracoxibe comparado com placebo nas primeiras 24 horas de pós-operatório
}

\author{
Evaluation of the analgesic effects of the lumiracoxib compared with placebo in \\ the 24 first postoperative hours
}

\author{
Vilmar Molon ${ }^{1}$; Cleber Dario Pinto Kruel²; Desiré Tarso Maioli3; Juliana Zanrosso Caran ${ }^{3}$; Raquel Cristina Lovison ${ }^{3}$
}

\author{
R E S U M O
}

\begin{abstract}
Objetivo: Testar o lumiracoxibe, utilizado preemptivamente contra um placebo, e analisar o consumo de analgésicos (dipirona e tramadol) nas primeiras $24 \mathrm{~h}$ do pós-operatório. Métodos: Sessenta pacientes submetidas à mamoplastia de aumento, sob anestesia peridural, foram selecionadas e divididas de forma randomizada em dois grupos - Gl e GIl. Sessenta minutos antes da operação, por via oral, as 30 pacientes do Gl receberam um comprimido de lumiracoxibe $400 \mathrm{mg}$, e as 30 do Gll um comprimido de placebo. A dor pós-operatória foi avaliada através da escala analógica visual, graduada de 0 (sem dor) a 10 (dor de maior intensidade), sendo aplicada sempre que houvesse referência de dor. Para escores obtidos iguais ou menores que 4, a paciente recebia $15 \mathrm{mg} / \mathrm{kg}$ de dipirona, até máximo de um grama, via venosa. Para escores de 5, 6 ou 7, recebia $50 \mathrm{mg}$ de tramadol por via venosa. Escores de 8 ou mais eram tratados com $0,5 \mathrm{mg} / \mathrm{kg}$ de meperidina endovenosa. Resultados: Os escores obtidos no $\mathrm{Gl}$ foram significativamente menores que os do GIl dentro das primeiras 24 horas, resultando em consumo $20 \%$ menor de dipirona e 56\% menor de tramadol. Da mesma forma, observou-se que o tempo para solicitação de analgésicos pela primeira vez no pós-operatório foi de 191 minutos maior no Gl. Conclusão: O emprego do lumiracoxibe preemptivamente mostrou-se efetivo no combate à dor pós-operatória, reduzindo consideravelmente o consumo global de analgésicos nesse período e aumentando o tempo de solicitação de analgésico pela primeira vez.
\end{abstract}

Descritores: Analgesia. Procedimentos cirúrgicos ambulatórios. Período pós-operatório.

\section{INTRODUÇÃO}

A partir dos anos 60 houve vertiginosa expansão da cirurgia ambulatorial em todas as áreas cirúrgicas, expansão esta pressionada por fatores econômicos, avanço tecnológico de equipamentos cirúrgicos, novas técnicas bem como pelas novas drogas anestésicas com perfil farmacocinético e farmacodinâmico ideal para procedimentos ambulatoriais'.

Os procedimentos cirúrgicos minimamente invasivos (via endoscópica), por sua vez, diminuíram o trauma cirúrgico e muito auxiliaram na recuperação e alta precoce dos pacientes ${ }^{2}$. Na virada do século, os procedimentos cirúrgicos ambulatoriais superavam $70 \%{ }^{3}$. Eles também serviram para diminuir a incidência de infecção pós-operatória e o estresse do paciente, além de tornarem-se práticos tanto para o paciente como para a equipe cirúrgica ${ }^{4}$. No entanto, a alta precoce do paciente submetido passa pelo contorno de algumas variáveis, dentre as quais o controle da dor pós-operatória bem como o das náuseas e vômitos $^{5}$. Estes, são adequadamente controlados com fármacos como o ondansetron, metoclopramida, dexametasona e antihistamínicos, isolados ou em associação ${ }^{6}$. A dor, no entanto, tem-se mostrado como o fator mais importante a ser considerado, sendo que seu manejo inadequado propicia aumento do número de casos de hospitalização de pacientes previamente selecionados para procedimentos ambulatoriais ${ }^{7-10}$.

A literatura mostra diferentes formas de abordagem para o tratamento da dor pós-operatória. Elas podem ser englobadas em três principais: a) emprego de técnicas de anestesia regional, em suas variadas formas; b) clássica analgesia multimodal associando drogas analgésicas com mecanismos de ação diferentes e (c) analgesia preemptiva cujo conceito moderno postulado por Kissin"1 diz ser ela "um tratamento antinociceptivo que previne o estabelecimento de alterações no processamento central dos impulsos aferentes produzidos pelas lesões". A validade da analgesia preemptiva no combate à dor pós-operatória é muito controversa dentro da literatura médica, alguns demonstrando sua eficácia11,12, e outros não ${ }^{13}$.

Trabalho realizado na Universidade de Caxias do Sul e Universidade Federal do Rio Grande do Sul, Porto Alegre, RS, Brasil.

1 Professor Adjunto da Facudade de Medicina da Universidade de Caxias do Sul - Caxias do Sul, RS, Brasil; 2 Professor Asssociado da Faculdade de Medicina da UFRGS - Porto Alegre - RS - BR; 3 Acadêmicos de Medicina da Universidade de Caxias do Sul, RS, Brasil. 
Muitos fármacos têm sido utilizados e estudados ao longo do tempo com a finalidade de abolir, de forma preemptiva, a dor pós-operatória, mas nenhum grupo farmacológico foi e ainda é tão estudado como os AINEs, notadamente os inibidores seletivos da COX 2 (coxibs) ${ }^{14-16}$.

O presente estudo visou observar o comportamento do lumiracoxibe, inibidor seletivo da COX2, contra placebo utilizado preemptivamente em pacientes submetidas à mamoplastia de aumento sob anestesia peridural.

\section{METODO}

O presente estudo foi aprovado pelo Comitê de Ética e Pesquisa do Hospital do Círculo de Caxias do Sul Rio Grande do Sul - Brasil e executado conforme suas determinações éticas. Todas as pacientes participantes do presente estudo assinaram o Termo de Consentimento Livre e Informado, após o detalhamento da pesquisa e a concordância das mesmas. As pacientes, antes da assinatura do termo, foram submetidas à rigorosa avaliação préanestésica e somente as que se enquadraram dentro dos critérios de inclusão foram convidadas a participar do estudo.

Das pacientes avaliadas, foram selecionadas 60 , na faixa etária entre 18 e 57 anos, estado físico ASA I e II, sem distinção de raça ou classe social, tendo em comum apenas o procedimento cirúrgico (mamoplastia de aumento) e a técnica anestésica que seriam submetidas (bloqueio peridural). As pacientes foram divididas de forma randomizada em dois grupos de 30. O Grupo I (GI) recebeu, em tempo entre 45 e 60 minutos antes do início do ato anestésico, um comprimido de lumiracoxibe $400 \mathrm{mg}$, enquanto que o Grupo II (GII) recebeu um comprimido morfologicamente idêntico de placebo também em período de 45 a 60 minutos antes da aplicação da anestesia.

Tanto o pesquisador como o cirurgião e a enfermagem em contato com a paciente desconheciam qual medicação fora ingerida. Apenas uma pessoa, fora do grupo de estudo e que era responsável pela administração da medicação, tinha conhecimento do que estava sendo administrado às pacientes.

Os dados demográficos de ambos os grupos são vistos na tabela 1.

Foram excluídas as pacientes que se situavam fora da faixa etária selecionada (18-57 anos); as que pre-

Tabela 1 - Dados Demográficos e Estado Físico (ASA).

\begin{tabular}{lcc}
\hline & $\begin{array}{c}\text { Grupo I } \\
(\mathrm{n}=30)\end{array}$ & $\begin{array}{c}\text { Grupo II } \\
(\mathrm{n}=30)\end{array}$ \\
\hline Idade (anos) & $29 \pm 7$ & $33 \pm 13$ \\
Peso (kg) & $55 \pm 6,6$ & $56 \pm 6,3$ \\
Altura (cm) & $165 \pm 5$ & $165 \pm 5$ \\
Estado Físico ASA(I/II) & $25 / 5$ & $25 / 5$ \\
\hline
\end{tabular}

Valores de média \pm desvio padrão. feriram anestesia geral; as que apresentavam história de hipersensibilidade aos AINEs e/ou coxibs; as com história de asma brônquica ou outras doenças pulmonares obstrutivas; as com história de doença ácido-péptica pregressa ou em curso; as portadoras de cardiopatia isquêmica e/ou hipertensiva e as com insuficiência renal.

A randomização foi feita através de envelopes lacrados e numerados por pessoa fora do grupo de estudo e de atividade profissional fora do ambiente hospitalar. Esta pessoa recebeu 60 pequenos envelopes opacos dentro de uma caixa fechada contendo 30 comprimidos de lumiracoxibe $400 \mathrm{mg}$ e 30 de placebo e uma folha com numeração de 1 a 60 . Tomando um comprido qualquer à mão, retirava um envelope aleatoriamente da urna e colocava o comprimido em seu interior, fechando-o imediatamente e a seguir anotava na folha, ao lado do número correspondente ao envelope sorteado, a substância contida nele. O procedimento foi repetido até terem sido preparados todos os envelopes e a folha numerada preenchida. Na seqüência, a folha foi colocada em um envelope lacrado e entregue ao pesquisador e, da mesma forma, a urna fechada, contendo os envelopes com os comprimidos.

A cada ato cirúrgico programado, o pesquisador levava a urna ao hospital onde uma pessoa da enfermagem, mas não envolvida ao procedimento, retirava aleatoriamente um envelope e administrava seu conteúdo à paciente, devolvendo-o vazio, sendo posteriormente anexado ao prontuário.

Todas as pacientes foram submetidas a bloqueio peridural torácico nos interespaços $\mathrm{T}_{5}$ a $\mathrm{T}_{8}$ optando-se por aquele que se mostrasse mais acessível.

Previamente ao bloqueio, instalava-se uma via venosa e iniciava-se infusão de solução de Ringer Lactato $7 \mathrm{~mL} / \mathrm{kg} / \mathrm{h}$ contínua ao longo de todo o procedimento. Seguia-se monitorização e instalação de cateter nasal de oxigênio e posicionamento em decúbito lateral esquerdo. Neste momento, a paciente era sedada com 3 a 4,5mg de midazolam endovenoso. Seguia-se o procedimento de punção do espaço peridural. Após a injeção do anestésico, introduzia-se cateter peridural e reposicionava-se a paciente em decúbito dorsal mantendo-a sedada com doses intermitentes de midazolam. Eventuais episódios de hipotensão eram corrigidos com doses fracionadas de efedrina, procurando-se manter os níveis tensionais nunca abaixo de 1/4 dos valores prévios ao bloqueio. O anestésico utilizado em todas foi a ropivacaína $0,7 \%$, (concentração preparada a partir da ropivacaína $0,75 \%$ ) em volume de $0,5 \mathrm{~mL} / \mathrm{kg}$ $(3,5 \mathrm{mg} / \mathrm{kg})$ tendo como limite máximo total de $230 \mathrm{mg}$.

Ao longo do procedimento foram medidos os seguintes tempos:

- $t_{0}=$ ingestão do comprimido (lumiracoxibe ou placebo);

$-t_{1}=$ bloqueio espinhal;

- $t_{2}=$ início da operação;

- $t_{3}=$ término da operação;

- $t_{4}=$ solicitação de analgésico no pós-operatório pela primeira vez. 
A análise do desfecho foi realizada através da medida do tempo entre a ingestão do comprimido e a solicitação de analgésico pela primeira vez no pós-operatório, além da análise do consumo de analgésicos nas primeiras $24 \mathrm{~h}$ - tempo que equivale à duração de ação do lumiracoxibe.

Os analgésicos empregados para o tratamento da dor pós-operatória foram a dipirona, tramadol e meperidina. O critério estabelecido para o emprego delas seguiu a escada analgésica proposta pela Organização Mundial da Saúde (OMS) para o tratamento da dor, que preconiza drogas não opióides, como a dipirona, para o tratamento da dor de pequena intensidade.

Para dores de moderada intensidade, a OMS propõe associarem-se às drogas não opióides, os opióides de moderada ação agonista no qual se enquadra o tramadol. Por fim, para dor severa esta escala propõe o uso de opióides de ação agonista intensa que aqui é representado pela meperidina. A utilização de cada uma dessas drogas era determinada pelo escore obtido através da aplicação da escala analógica visual da dor (EAV) sempre que a paciente referisse dor ou desconforto e que desejasse receber analgésico.

Sempre que fosse solicitado analgésico e, ao ser confrontada, a EAV indicasse valores de 4 ou menos era administrado dipirona $15 \mathrm{mg} / \mathrm{kg}$ via venosa até o máximo de 1 grama. Decorridos 30 minutos da ingestão do medicamento a paciente era novamente submetida à escala. Se os valores se mantivessem iguais ou aumentassem, recebia tramadol $50 \mathrm{mg}$ via venosa. Por outro lado, na confrontação da dor com a EAV fossem obtidos valores de 5, 6 ou 7 , já recebia tramadol $50 \mathrm{mg}$ via venosa. A seguir e após 30 minutos se estes valores se mantivessem iguais ou maiores, recebia $0,5 \mathrm{mg} / \mathrm{kg}$ de meperidina. Se o valor referido na EAV fosse de 4 ou menos recebia $15 \mathrm{mg} / \mathrm{kg}$ de dipirona. Por fim, se a paciente referisse intensidade dolorosa igual ou maior a 8 , recebia de imediato meperidina $0,5 \mathrm{mg} / \mathrm{kg}$. Após 30 minutos, se a dor cedesse mas ainda a paciente solicitasse analgésico, este seria administrado de acordo com o valor referido na EAV pela paciente.

Na sala de recuperação pós-anestésica, a EAV era manipulada por pessoa treinada para tal, geralmente a enfermeira-chefe. A paciente lá permanecia por período nunca inferior a cinco horas e, ao ter alta, recebia uma EAV e um protocolo para uso da medicação analgésica em sua residência. Ambos haviam sido exaustivamente explicados a ela pelo pesquisador já na entrevista pré-anestésica $\mathrm{e}$, neste momento, eram reforçados os mesmos esclarecimentos pela enfermeira. O protocolo deveria ser devolvido ao pesquisador após o fechamento das primeiras 24 horas de pós-operatório. A medicação a ser utilizada após a alta era exatamente a mesma utilizada no hospital, havendo somente a conversão para via oral. A meperidina não era prescrita para uso após a alta, sendo que, caso a paciente referisse episódio de dor e a EAV assinalasse 8 ou mais, ela deveria procurar a equipe médica.

Os critérios utilizados para a alta hospitalar foram os seguintes: a) ausência de efeitos residuais do bloqueio anestésico; b) paciente referisse valores na EAV inferiores a $3 ;$ c) ausência de náuseas e vômitos; d) sinais vitais estáveis e saturação da hemoglobina superior a 95\%.

As variáveis não paramétricas foram analisadas pelo teste $U$ de Mann Whitney, enquanto que as paramétricas foram analisadas pelo teste t de Student. O período em que as pacientes estiveram livres do uso de analgésicos foi analisado através de Curva de Kaplan-Meyer e a comparação entre os grupos pelo teste de Log Rank. $\mathrm{O}$ nível de significância adotado foi de á =0,05 e o poder de estudo de $70 \%$. Os dados foram analisados com o auxílio do programa SPSS, versão 11.0.

\section{RESULTADOS}

Nenhuma das pacientes envolvidas no estudo necessitou utilizar meperidina. Também não houve perda de nenhuma paciente, por qualquer razão, ao longo da pesquisa. Os dados demográficos, bem como o estado físico das pacientes mostrou paridade entre os grupos (Tabela 1).

Da mesma forma, os dados gerais dos grupos, no que concerne ao volume anestésico empregado e os tempos medidos, apontaram homogeneidade (Tabela 2).

Os detalhes da analgesia podem ser visto na tabela 3. Vê-se boa efetividade no tratamento da dor quan-

Tabela 2 - Dados gerais da amostra dividida em dois grupos.

\begin{tabular}{|c|c|c|c|c|c|c|}
\hline & Tratamento & $\mathrm{N}$ & Média & $d p$ & $\begin{array}{l}\text { Diferença } \\
\text { das médias }\end{array}$ & $p$ \\
\hline \multirow[t]{2}{*}{ Volume de anestésico injetado (ml) } & Lumiracoxibe & 30 & 26 & 2,97 & 0,43 & 0,59 \\
\hline & Placebo & 30 & 26 & 3,24 & & \\
\hline \multirow{2}{*}{$\begin{array}{l}\text { Tempo entre a ingestão e o início } \\
\text { do bloqueio (min) }\end{array}$} & Lumiracoxibe & 30 & 39 & 10,85 & 0,43 & 0,90 \\
\hline & Placebo & 30 & 40 & 16,80 & & \\
\hline \multirow{2}{*}{$\begin{array}{l}\text { Tempo entre a ingestão e o início } \\
\text { da cirurgia (min) }\end{array}$} & Lumiracoxibe & 30 & 59 & 12,42 & 2,66 & 0,47 \\
\hline & Placebo & 30 & 61 & 15,82 & & \\
\hline \multirow[t]{2}{*}{ Tempo de duração da operação (min) } & Lumiracoxibe & 30 & 88 & 30,29 & 3,26 & 0,69 \\
\hline & Placebo & 30 & 85 & 34,35 & & \\
\hline
\end{tabular}

$d p=$ desvio padrão 
Tabela 3 - Resultados do estudo.

\begin{tabular}{|c|c|c|c|c|c|c|}
\hline & Tratamento & $\mathrm{N}$ & Média & $d p$ & $\begin{array}{l}\text { Diferença } \\
\text { das médias }\end{array}$ & $p$ \\
\hline \multirow{2}{*}{$\begin{array}{l}\text { Tempo desde a realização do bloqueio } \\
\text { até a primeira dose de analgésico (min) }\end{array}$} & Lumiracoxibe & 30 & 535 & 387,93 & \multirow[t]{2}{*}{191,72} & \multirow[t]{2}{*}{0,02} \\
\hline & Placebo & 30 & 344 & 240,38 & & \\
\hline \multirow{2}{*}{$\begin{array}{l}\text { Número de tomadas de analgésicos nas } \\
\text { primeiras } 24 \text { horas pós-bloqueio }\end{array}$} & Lumiracoxibe & 30 & 2,17 & 1,34 & \multirow[t]{2}{*}{1,03} & \multirow[t]{2}{*}{0,005} \\
\hline & Placebo & 30 & 3,2 & 1,40 & & \\
\hline \multirow{2}{*}{$\begin{array}{l}\text { Número de vezes de uso de dipirona } \\
\text { no primeiro dia }\end{array}$} & Lumiracoxibe & 30 & 1,7 & 1,17 & \multirow[t]{2}{*}{0,43} & \multirow[t]{2}{*}{0,12} \\
\hline & Placebo & 30 & 2,13 & 0,97 & & \\
\hline \multirow{2}{*}{$\begin{array}{l}\text { Número de vezes de uso de tramadol } \\
\text { no primeiro dia }\end{array}$} & Lumiracoxibe & 30 & 0,47 & 0,73 & \multirow[t]{2}{*}{0,6} & \multirow[t]{2}{*}{0,02} \\
\hline & Placebo & 30 & 1,07 & 1,20 & & \\
\hline
\end{tabular}

do o lumiracoxibe foi utilizado preemptivamente. Todos os parâmetros pesquisados, à exceção do consumo de dipirona no primeiro dia, mostraram significância estatística.

Estes mesmos resultados, testados através da curva de Kaplan-Meyer, também mostraram diferença significativa no tempo livre de necessidade de analgésicos que, para o $\mathrm{Gl}$, foi de 415 minutos com intervalo de confiança estabelecido entre 237 e 593 minutos, e para o Gll este tempo foi de 298 minutos com intervalo de confiança situado entre 269 e 326 minutos. O teste de Log Rank mostrou p=0,01.

Da mesma forma, os efeitos colaterais e complicações levantadas ao longo de todo o estudo não mostraram diferenças entre os grupos.

\section{DISCUSSÃO}

A polêmica sobre a efetividade da analgesia preemptiva no manejo da dor pós-operatória se mantém viva e controversa. Os fármacos testados para esta finalidade têm sido os mais diversos e dentre esses estão os opióides, bloqueio peridural, antagonistas dos receptores NMDA, bloqueios anestésicos periféricos, AINEs e os coxibs.

Especificamente em relação aos AINEs, seu emprego está relacionado a episódios de hemorragia digestiva, insuficiência renal, além de alterações da coagulação sanguínea ${ }^{17}$. Os coxibs, por sua vez, mostram-se mais seguros com relação ao desenvolvimento desses paraefeitos e, por isso, têm sido utilizados de forma crescente para o tratamento da dor pós-operatória, seja de forma preemptiva, seja na terapêutica multimodal clássica ${ }^{18}$.

O lumiracoxibe é inibidor seletivo da COX-2 desenvolvido primeiramente para o tratamento da osteoartrite, da artrite reumatóide e da dor aguda ${ }^{19}$. Ele é quimicamente diferenciado dos demais coxibs por apresentar em sua estrutura um grupamento ácido que Ihe confere propriedades com esta característica, como rápida absorção a partir do intestino com alta e sustentada concentração no líquido sinovial20.

Embora sua concentração plasmática decline com certa rapidez, em média 4 horas ${ }^{21}$, o lumiracoxibe apresenta trofismo grande pelos tecidos inflamados, resultando, com isso, em analgesia sustentada ${ }^{22}$.
Muito embora não haja estudos na literatura médica mostrando o uso preemptivo do lumiracoxibe para o tratamento da dor pós-operatória, Bombardier et al. ${ }^{23}$ mostraram que todos os inibidores seletivos da COX-2 possuem eficácia semelhante aos AINEs no que tange ao combate à dor pós-operatória. Os coxibs, no entanto, apresentam grande vantagem sobre os AINEs no que se refere à proteção gástrica, principalmente em tratamentos prolongados $^{24,25}$.

O resultado do presente estudo mostrou desempenho efetivo desse fármaco no tratamento da dor pósoperatória. Em relação ao tempo, para a solicitação de analgésicos pela primeira vez no pós-operatório, a diferença média entre os dois grupos foi significativa e de 191 minutos.

Ong et al. ${ }^{26}$, em metanálise publicada, levantou na literatura 17 estudos randomizados que avaliavam o emprego pré-operatório de AINEs versus seu emprego no pós-operatório ${ }^{26}$. Deles, cinco mostraram diferenças significativas favoráveis ao pré-tratamento com relação ao tempo para solicitação de analgésicos pela primeira vez no pós-operatório ${ }^{27}$, o que vem corroborar as conclusões obtidas no presente estudo com relação a esse item.

Também Romsing ${ }^{25}$, em revisão sistemática, mostram que todos os coxibs aumentam significativamente o tempo de solicitação de analgésico no pós-operatório pela primeira vez, chegando ao extremo de até 23 horas $^{28}$.

Por outro lado, Moiniche ${ }^{13}$ em sua ampla revisão sistemática sobre as diversas modalidades de analgesia preemptiva, analisando 20 estudos sobre os AINEs, não conseguiu encontrar relação entre o emprego desses fármacos premptivamente e melhora dos níveis da dor pósoperatória ${ }^{29}$. Cinco estudos analisaram o tempo para a primeira solicitação analgésica no pós-operatório e, destes, apenas dois mostraram significância estatística no sentido de aumentar tempo. Em um, houve aumento de $90^{30} \mathrm{e}$, em outro de 49 minutos $^{31}$.

No presente estudo, por sua vez, o suplemento analgésico necessário nas primeiras 24 horas de pós-operatório foi significativamente diferente entre GI e GII. A diferença de consumo da dipirona entre eles foi de apenas 20. Porém, quando analisado o consumo de tramadol, a dife- 
rença aumenta entre os grupos. Essa diferença representa consumo 56\% menor de tramadol pró Gl.

Ong et al. ${ }^{26}$, mostraram em sua metanálise que, dos 12 estudos em que houve comparação entre o uso préincisional e pós-incisional dos AINEs, oito mostraram resultados amplamente favoráveis ao uso ${ }^{32}$ com relação ao consumo de analgésicos no pós-operatório, vindo ao encontro, também, dos resultados obtidos no presente estudo.

A diminuição acentuada no consumo de analgésicos no pós-operatório também é demonstrada na revisão sistemática de Romsing ${ }^{25}$, sendo que em um estudo há diminuição de $83 \%$ no consumo de analgésicos nas primeiras 24 horas $^{33}$.

Essas constatações vêm ao encontro aos resultados obtidos neste estudo, no qual ficou evidenciado aumento de 191 minutos no tempo para solicitação de analgésicos pela primeira vez e diminuição de $53 \%$ no consumo de tramadol nas primeiras 24 horas do pós-operatório.

Da mesma forma, a revisão sistemática publicada por Straube ${ }^{34}$ mostra evidências claras do benefício dos coxibs utilizados preemptivamente no alívio da dor pós-operatória em 75\% dos estudos, diminuindo consideravelmente o consumo de analgésicos e aumentando a satisfação do paciente.

Moiniche ${ }^{13}$, por sua vez, em sua revisão sistemática também não conseguiu mostrar redução significativa no consumo de analgésicos no pós-operatório quando os AINEs haviam sido utilizados preemptivamente. Dos 20 estudos incluídos nesta revisão, em apenas dois deles havia diminuição significativa no consumo de analgésicos no pós-operatório ${ }^{35,36}$.

Apesar de haver sido retirado recentemente por haver suspeita de que o lumiracoxibe possa causar lesões hepáticas, muitos trabalhos tem mostrado que seu emprego é seguro mesmo a longo prazo no tratamento de doenças crônicas como a osteoartrite principalmente quando comparado com outros inibidores seletivos da COX-2 ${ }^{37-39}$

Em conclusão, a administração de dose única, via oral, de $400 \mathrm{mg}$ mostrou-se efetiva em diminuir o consumo de analgésicos por diminuir a dor pós-operatória em pacientes submetidas à mamoplastia de aumento sob anestesia peridural.

\title{
A B S T R A C T
}

\begin{abstract}
Objectives: To test lumiracoxib used preemptively against a placebo, and to analyse the analgesic administration (dipirona and tramadol) in the 24 first postoperative hours. Methods: Sixty patients undergone to a breast aumentation, under epidural anesthesia, were selected and put randomly in two groups - Gl and Gll. Sixty minutes before the surgery, it was administered orally, one pill of lumiracoxib $400 \mathrm{mg}$ to the $30 \mathrm{Gl}$ patients and one pill of placebo to the $30 \mathrm{Gll}$ patients. The postoperative pain was evaluated through the visual analogic scale, ranged from 0 (painless) to 10 (most intensive pain), being applied to the patient whenever having pain. For the same scores or under 4, it was administered intravenously $15 \mathrm{mg} / \mathrm{kg}$ of dipirona up to a maximum of 1g. For scores of 5, 6 or 7, the patient had intravenously 50mg of tramadol. Scores 8 or more were treated intravenously with 0,5 $\mathrm{mg} / \mathrm{kg}$ of meperidine. Results: The scores in Gl were significantly lower than the ones obtained in Gll within the first 24 hours, with the need of $20 \%$ less dipirona and $56 \%$ of tramadol in this period of time. In the same way, it was observed that analgesic solicitation for the first time had a delay of 191 minutes in Gl in comparison to GII. Conclusion: The use of lumiracoxib preemptively, was effective in the treatment of postoperative pain, reducing considerably the consume of analgesics, and enlarging the time for the first medication.
\end{abstract}

Key words: Analgesia. Ambulatory surgical procedures. Postoperative period.

\section{REFERENCIAS}

1. Burden N. Outpatient surgery: a view through history. J Perianesth Nurs. 2005; 20(6):435-7.

2. Pandit SK. Ambulatory anesthesia and surgery in America: a historical background and recent innovations. J Perianesth Nurs. 1999; 14(5):270-4.

3. Poole EL. Ambulatory surgery: the growth of an industry. J Perianesth Nurs. 1999; 14(4):201-6.

4. Pavlin DJ, Chen C, Penaloza DA, Polissar NL, Buckley FP. Pain as a factor complicating recovery and discharge after ambulatory surgery. Anesth Analg. 2002; 95(3):627-34.

5. Pavlin DJ, Chen C, Penaloza DA, Buckley FP. A survey of pain and other symptoms that affect the recovery process after discharge from an ambulatory surgery unit. J Clin Anesth. 2004; 16(3):200-6.

6. White PF. The role of non-opioid analgesic techniques in the management of pain after ambulatory surgery. Anesth Analg. 2002; 94(3):577-85.

7. McHugh GA, Thoms GM. The management of pain following daycase surgery. Anaesthesia. 2002; 57(3):270-5.
8. Rocchi A, Chung F, Forte L. Canadian survey of postsurgical pain and pain medication experiences. Can J Anaesth. 2002; 49(10):1053-6.

9. Mulroy MF, McDonald SB. Regional anesthesia for outpatient surgery. Anesthesiol Clin North America. 2003; 21(2):289-303.

10. Kehlet H, Dahl JB. The value of "multimodal" or "balanced analgesia" in postoperative pain treatment. Anesth Analg. 1993; 77(5):1048-56.

11. Kissin I. Preemptive analgesia at the crossroad. Anesth Analg. 2005; 100(3):754-6.

12. Pavlin DJ, Horvath KD, Pavlin EG, Sima K. Preincisional treatment to prevent pain after ambulatory hernia surgery. Anesth Analg. 2003; 97(6):1627-32.

13. Møiniche S, Kehlet H, Dahl JB. A qualitative and quantitative systematic review of preemptive analgesia for postoperative pain relief: the role of timing of analgesia. Anesthesiology. 2002; 96(3):725-41.

14. O'Hanlon DM, Thambipillai T, Colbert ST, Keane PW, Given HF. Timing of pre-emptive tenoxicam is important for postoperative analgesia. Can J Anaesth. 2001; 48(2):162-6. 
15. Karamanlio $\square$ lu B, Turan A, Memi $\square$ D, Türe M. Preoperative oral rofecoxib reduces postoperative pain and tramadol consumption in patients after abdominal hysterectomy. Anesth Analg. 2004; 98(4):1039-43.

16. Straube S, Derry S, McQuay HJ, Moore RA. Effect of preoperative Cox-II-selective NSAIDs (coxibs) on postoperative outcomes: a systematic review of randomized studies. Acta Anaesthesiol Scand. 2005; 49(5):601-13.

17. Kam PCA, Power I. New selective COX-2 inhibitors. Pain Rev. 2000; $7(1): 3-13$.

18. Chaiamnuay S, Allison JJ, Curtis JR. Risks versus benefits of cyclooxygenase-2-selective nonsteroidal antiinflammatory drugs. Am J Health Syst Pharm. 2006; 63(19):1837-51.

19. Marshall PJ, Berry JC, Wasvary J, van Duzer J, Du Z, Scott G, Rordorf C, Milosavljev S, Fujimoto RA. The in vitro and in vivo selectivity of COX 189, a new and highly seletive inhibitor of COX2 [Abstract SAT0013]. Ann Rheum Dis. 61 (Suppl. 1):259.

20. Day RO, McLachlan AJ, Graham GG, Williams KM. Pharmacokinetics of nonsteroidal anti-inflammatory drugs in synovial fluid. Clin Pharmacokinet. 1999; 36(3):191-210.

21. Rainsford KD, Schweitzer A, Brune K. Autoradiographic and biochemical observations on the distribution of non-steroid antiinflammatory drugs. Arch Int Pharmacodyn Ther. 1981; 250(2):18094.

22. Rordorf CM, Choi L, Marshall P, Mangold JB. Clinical pharmacology of lumiracoxib: a selective cyclo-oxygenase-2 inhibitor. Clin Pharmacokinet. 2005; 44(12):1247-66.

23. Bombardier $C$, Laine $L$, Reicin A, Shapiro D, Burgos-Vargas R, Davis B, Day R, Ferraz MB, Hawkey CJ, Hochberg MC, Kvien TK, Schnitzer TJ; VIGOR Study Group. Comparison of upper gastrointestinal toxicity of rofecoxib and naproxen in patients with rheumatoid arthritis. VIGOR Study Group. N Engl J Med. 2000; 343(21):1520$8,2 \mathrm{p}$ following 1528

24. Goldstein JL, Correa P, Zhao WW, Burr AM, Hubbard RC, Verburg KM, Geis GS. Reduced incidence of gastroduodenal ulcers with celecoxib, a novel cyclooxygenase-2 inhibitor, compared to naproxen in patients with arthritis. Am J Gastroenterol. 2001; 96(4):1019-27.

25. Rømsing J, Møiniche S. A systematic review of COX-2 inhibitors compared with traditional NSAIDs, or different COX-2 inhibitors for post-operative pain. Acta Anaesthesiol Scand. 2004; 48(5):52546.

26. Ong CK, Lirk P, Seymour RA, Jenkins BJ. The efficacy of preemptive analgesia for acute postoperative pain management: a metaanalysis. Anesth Analg. 2005; 100(3):757-73.

27. Ong KS, Seymour RA, Chen FG, Ho VC. Preoperative ketorolac has a preemptive effect for postoperative third molar surgical pain. Int J Oral Maxillofac Surg. 2004; 33(8):771-6.

28. Reuben SS, Bhopatkar S, Maciolek H, Joshi W, Sklar J. The preemptive analgesic effect of rofecoxib after ambulatory arthroscopic knee surgery. Anesth Analg. 2002; 4(1):55-9.
29. Priya V, Divatia JV, Sareen R, Upadhye S. Efficacy of intravenous ketoprofen for pre-emptive analgesia. J Postgrad Med. 2002; 8(2):109-12.

30. Colbert ST, O'Hanlon DM, McDonnell C, Given FH, Keane PW. Analgesia in day case breast biopsy-the value of pre-emptive tenoxicam. Can J Anaesth. 1998; 5(3):217-22.

31. O'Hanlon JJ, Muldoon T, Lowry D, McCleane G. Improved postoperative analgesia with preoperative piroxicam. Can J Anaesth. 1996; 43(2):102-5.

32. Daniels SE, Desjardins PJ, Talwalker S, Recker DP, Verburg KM. The analgesic efficacy of valdecoxib vs. oxycodone/acetaminophen after oral surgery. J Am Dent Assoc. 2002; 133(5):611-21.

33. Nordbladh I, Ohlander B, Björkman R. Analgesia in tonsillectomy: a double-blind study on pre and post-operative treatment with diclofenac. Clin Otolaryngol Allied Sci. 1991; 16(6):554-8.

34. Likar R, Krumpholz R, Pipam W, Sadjak A, Kapral S, Forsthuber E, Bernatzky G, List FW. [Randomized, double-blind study with ketoprofen in gynecologic patients. Preemptive analgesia study following the Brevik-Stubhaug design]. Anaesthesist. 1998; 47(4):303-10.

35. Colbert ST, O'Hanlon DM, McDonnell C, Given FH, Keane PW. Analgesia in day case breast biopsy-the value of pre-emptive tenoxicam. Can J Anaesth. 1998; 45(3):217-22.

36. Rosaeg OP, Krepski B, Cicutti N, Dennehy KC, Lui AC, Johnson DH. Effect of preemptive multimodal analgesia for arthroscopic knee ligament repair. Reg Anesth Pain Med. 2001; 26(2):125-30.

37. Issioui T, Klein KW, White PF, Watcha MF, Skrivanek GD, Jones SB, $\mathrm{Hu}$ J, Marple BF, Ing C. Cost-efficacy of rofecoxib versus acetaminophen for preventing pain after ambulatory surgery. Anesthesiology. 2002; 97(4):931-7.

38. Fletcher D, Zetlaoui P, Monin S, Bombart M, Samii K. Influence of timing on the analgesic effect of intravenous ketorolac after orthopedic surgery. Pain. 1995; 61(2):291-7.

39. Fleischmann R, Tannenbaum H, Patel NP, Notter M, Sallstig P, Reginster JY. Long-term retention on treatment with lumiracoxib $100 \mathrm{mg}$ once or twice daily compared with celecoxib $200 \mathrm{mg}$ once daily: a randomised controlled trial in patients with osteoarthritis. BMC Musculoskelet Disord. 2008; 9:32.

Recebido em 20/08/2008

Aceito para publicação em 22/10/2008

Conflito de interesse: nenhum

Fonte de financiamento: nenhuma

\section{Como citar este artigo:}

Molon V, Kruel CDP, Maioli DT, Caran JZ, Lovison RC. Avaliação do efeito analgésico preemptivo do lumiracoxibe comparado com placebo nas primeiras 24 horas de pós-operatório. Rev Col Bras Cir. [periódico na Internet] 2009; 36(1). Disponível em URL: http://www.scielo.br/rcbc

\section{Endereço para correspondência:}

Cleber Dario Pinto Kruel

E-mail: cdkruel@yahoo.com.br 